\title{
Variación temporal de la diversidad de estafilínidos (Coleoptera: Staphylinidae) nocturnos en un bosque tropical caducifolio de México
}

\author{
Temporal variation of the diversity of nocturnal staphylinids (Coleoptera: Staphylinidae) in a \\ tropical dry forest in Mexico
}

\author{
Esteban Jiménez-Sánchez ${ }^{1 *}$, Santiago Zaragoza-Caballero² y Felipe A. Noguera ${ }^{3}$ \\ ${ }^{1}$ Laboratorio de Zoología, FES-Iztacala, Universidad Nacional Autónoma de México. Apartado postal 314, 54090, Tlalnepantla, Estado de México, \\ México. \\ ${ }^{2}$ Departamento de Zoología, Instituto de Biología, Universidad Nacional Autónoma de México. Apartado postal 70-153, 04510, México, D. F., \\ México. \\ ${ }^{3}$ Estación de Biología Chamela, Instituto de Biología, Universidad Nacional Autónoma de México. Apartado postal 21, 48980, San Patricio, Jalisco, \\ México. \\ *Correspondencia: estjimsan@yahoo.com.mx
}

\begin{abstract}
Resumen. Se estudió la variación temporal de los estafilínidos nocturnos en la sierra de Huautla, Morelos, México. El estudio se realizó entre noviembre de 1995 y octubre de 1996, los muestreos fueron mensuales y se utilizaron trampas de luz como método de muestreo. Se recolectaron 14886 individuos de 91 especies, 49 géneros, 15 tribus y 9 subfamilias. Las subfamilias más abundantes y ricas en especies fueron Paederinae, Aleocharinae y Oxytelinae. La distribución de individuos por especie fue heterogénea, con pocas especies muy abundantes y muchas con pocos individuos. Hubo diferencias estacionales en riqueza y abundancia y los valores más altos se registraron en la época de lluvias. La abundancia y la riqueza estuvieron relacionadas con la precipitación, pero no con la temperatura.
\end{abstract}

Palabras clave: Staphylinidae, riqueza, abundancia, variación temporal, bosque tropical caducifolio.

\begin{abstract}
A study of the temporal variation of the nocturnal staphylinids from Sierra de Huautla, Morelos, Mexico is presented. The fieldwork was carried out between November 1995 and October 1996, with monthly light trap samplings used as collection method. A total of 14886 individuals were collected, belonging to 91 species, 49 genera, 15 tribes and 9 subfamilies. The subfamilies with the greatest number of individuals and species were Paederinae, Aleocharinae and Oxytelinae. The distribution of individuals per species was heterogeneous, with few species very abundant and many with only a few individuals. There were seasonal differences in richness and abundance and the highest number of species and individuals were recorded during the rainy season. The abundance and richness were related with precipitation, but not with temperature.
\end{abstract}

Key words: Staphylinidae, richness, abundance, temporal variation, Tropical Dry Forest.

\section{Introducción}

La actividad estacional de los insectos es un fenómeno que ha sido registrado muchas veces en ecosistemas tropicales donde hay una clara alternancia de una estación seca y una húmeda (Wolda, 1978). No obstante, esta estacionalidad puede variar de acuerdo al grupo taxonómico de que se trate y en general cada patrón representa una solución diferente de cada especie a las limitaciones locales de agua, alimento, fotoperiodo, temperatura, etc. (Pescador et al., 2002), dando lugar ello a una gran variedad de patrones temporales de diversidad

Recibido: 05 marzo 2007; aceptado: 06 julio 2008
(Kato et al., 1995). Considerando lo anterior, el presente trabajo tiene como objetivo determinar en un bosque tropical estacional la variación temporal en la diversidad de un grupo de coleópteros, con una gran diversidad de hábitos alimenticios y requerimientos de hábitats, como es Staphylinidae (Navarrete-Heredia et al., 2002).

\section{Materiales y métodos}

Área de estudio. El estudio se realizó en la porción centro sur de la sierra de Huautla, entre los $18^{\circ} 20^{\prime} 10^{\prime \prime}-18^{\circ} 34^{\prime}$ $20 " \mathrm{~N}$ y $98^{\circ} 51^{\prime} 20^{\prime \prime}-99^{\circ} 08^{\prime} 15^{\prime}$ O Órea que queda incluida parcialmente en la Reserva de la Biosfera Sierra de 
Huautla (Dorado et al., 2005). Los muestreos se realizaron alrededor de las antiguas instalaciones del Centro de Educación Ambiental Sierra de Huautla (CEAMISH) que se ubican $2.5 \mathrm{k} \mathrm{m}$ al norte y $4 \mathrm{~km}$ al oeste del poblado de Huautla $\left(18^{\circ} 27^{\prime} .671 \mathrm{~N}, 99^{\circ} 02^{\prime} .475 \mathrm{O}\right)$ y cuya altitud es de $900 \mathrm{~m}$. Dichas instalaciones están justo enfrente de la presa Lorenzo Vázquez, la cual se ubica en el cauce del río Quilamula, que tiene una orientación noroestesureste en esa porción. Este arroyo es temporal y lleva agua solamente durante la época de lluvias; sin embargo, después del reservorio el cauce del arroyo mantiene el flujo de agua todo el año, debido al drenado constante de dicho reservorio.

De acuerdo con la clasificación de Köppen modificada por García (1981), el clima de la región es templado subhumedo, tipo Awo"(w)(i')g. El promedio anual de precipitación para el periodo 1981-1997 fue de $824 \mathrm{~mm}$, con el $80 \%$ de las lluvias cayendo entre junio y septiembre. La temperatura promedio para el mismo periodo fue de $24.7^{\circ} \mathrm{C}$, con una temperatura promedio máxima de $34.3^{\circ}$ $\mathrm{C}$ y una temperatura promedio mínima de $15.0^{\circ} \mathrm{C}$. La temperatura más alta durante dicho periodo fue registrada en mayo $\left(36.5^{\circ} \mathrm{C}\right)$ y la más baja en febrero $\left(12.5^{\circ} \mathrm{C}\right)(\mathrm{CNA}$, 2000). La precipitación total durante el periodo de estudio fue de $850 \mathrm{~mm}$, con la temperatura más alta registrada en abril y la más baja en enero.

La vegetación dominante es el bosque tropical caducifolio (BTC) y se conocen 882 especies de plantas vasculares (Dorado, 1997). Los árboles dominantes en la región son Conzattia multiflora (Robinson) Standley, Lysiloma acapulcense (Kunth) Benth., L. divaricata (Jacq.) Macbr. (Leguminosae) y varias especies de Bursera (Burseraceae) y Ceiba (Bombacaceae) (Dorado, 1997). En los ríos y cañadas estrechas se presenta un bosque de galería conformado por árboles más altos que los del BTC, tales como Licania arborea Seem. (Chrysobalanaceae), Sapindus saponaria L. (Sapindaceae), Guazuma ulmifolia (Lam.) (Sterculiacea), Ficus petiolaris Kunth, F. tecolutensis (Liebm.) (Moraceae), Daphnopsis americana (Mill.) J. Johnston (Thymelaeceae), Enterolobium cyclocarpum (Jacq.) Griseb., Pithecellobium dulce (Roxb.) Benth. (Leguminosae), Astianthus viminalis (HBK.) Baillon (Bignoniaceae), Bursera grandifolia (Schdl.) Engl. (Burseraceae), Euphorbia fulva Staff. (Euphorbiacea) and Salix humboltiana Willd. (Salicaceae). En áreas alteradas hay asociaciones secundarias formadas por leguminosas espinosas, tales como Acacia farnesiana (L.) Willd., Pithecellobium acatlense Benth., Mimosa polyantha Benth., M. benthamii J. F. Macbr. y Eysenhardtia polystachya (Ortega) Sarg. (Pérez-Jiménez et al., 1992).

Método de recolecta y régimen de muestreo. Se utilizaron 3 trampas de luz como método de muestreo. Estas trampas estuvieron compuestas de 2 fuentes luminosas: un foco de vapor de mercurio y una trampa de luz tipo Minnesota (Southwood, 1966), compuesta de 2 focos de luz ultravioleta de 20 watts ( 1 sin filtro), colocados sobre un contenedor de $20 \mathrm{~cm}$ de diámetro. Ambas fuentes de luz se colocaron frente a una sábana blanca de 1.80 por $1.50 \mathrm{~m}$, que servía como pantalla reflectora. Como líquido conservador se utilizó alcohol al $70 \%$ y las muestras correspondieron a los individuos capturados exclusivamente en el contenedor de la trampa de luz tipo Minnesota.

Estos sistemas de trampeo fueron colocados en 3 sitios diferentes y permanecieron en el mismo lugar durante todo el periodo de muestreo. La trampa 1 se colocó en el costado sur de las antiguas instalaciones del CEAMISH y tuvo la misma orientación; las trampas 2 y 3 se colocaron en el margen este de la presa Lorenzo Vázquez (frente a dichas instalaciones), con la trampa 2 orientada hacia el oeste y la 3 hacia el noroeste. Las trampas operaron simultáneamente durante 4 horas cada día (de las 20:00 a las 24:00 horas en el horario de verano y de las 19:00 a las 23:00 horas el resto del año).

El muestreo se realizó entre noviembre de 1995 a octubre de 1996, fue mensual y comprendió un día de recolecta por mes (coincidiendo con la fase del cuarto menguante lunar).

Método de análisis. Los valores de riqueza y abundancia corresponden al número de especies e individuos registrados. La diversidad y uniformidad fue analizada usando el índice de Shannon y la similitud mediante el índice de Jaccard. Para comparar la abundancia y riqueza entre estaciones se realizó una prueba no paramétrica Mann-Whitney U (Zar, 1996).

Para el análisis de fenología, se consideró que la época de lluvias fue de mayo a noviembre y la de sequía de diciembre a abril. Esto se determinó con base en el registro de precipitaciones mayores a $15 \mathrm{~mm}$ durante el mes, debido a que en otra región con BTC se ha registrado que eventos menores son interceptados casi en un $100 \%$ por el dosel y que su efecto a escala de la comunidad es reducido (Cervantes, 1988).

La relación de la abundancia y la riqueza con la precipitación y la temperatura se determinó mediante una regresión simple. Para calcular la diversidad, uniformidad y similitud se utilizó el programa BioDiversityPro 2.0 (McAleece, 1997). Las pruebas no paramétricas se realizaron con el programa StatView.

Material de referencia. Todo el material está depositado en la Colección Nacional de Insectos del Instituto de Biología de la Universidad Nacional Autónoma de México (CNIN). 


\section{Resultados}

Se recolectaron 14886 individuos de 91 especies, 49 géneros, 15 tribus y 9 subfamilias. Del total de especies sólo 2 pudieron ser determinadas hasta especie, debido a que el resto pertenecen a grupos no resueltos taxonómicamente (Klimaszewski, 2000; Anderson y Ashe, 2000; Chatzimanolis et al., 2004). Se incluye una relación de las mismas en el Apéndice 1.

Las subfamilias con mayor número de especies fueron Paederinae con 36, Aleocharinae con 25 y Oxytelinae con 14 (Cuadro 1), mientras que los géneros más diversos fueron Atheta con 7 especies, Lithocharis con 6, Homaeotarsus con 5 y Thinobius, Coproporus y Zyras con 4, respectivamente. Las subfamilias con mayor número de individuos fueron Oxytelinae con 11681 (78.4\%), Paederinae con 1440 (9.6\%) y Aleocharinae con 1376 (9.2\%) (Cuadro 1). La distribución de individuos por especie fue muy heterogénea, aunque prevalecieron las especies con pocos individuos (Fig. 1). Las especies más abundantes fueron Carpelimus sp. 1 con 5661 (38\%), Apocellus sp. con 3003 (20\%), Carpelimus sp. 2 con 1805 (12\%) y Platysthetus sp. con 592 (4\%); estas 4 especies conjuntaron el 74\% del total de los individuos registrados y pertenecen a la subfamilia Oxytelinae. En cambio, el $57 \%$ de las especies estuvieron representadas por menos de 10 individuos.

Variación temporal. La riqueza y abundancia variaron temporalmente y presentaron sus valores más altos en la época de lluvias (Figs. 2 y 3). En el caso de la riqueza, el patrón registrado no fue estacional, observándose 3 incrementos importantes a lo largo del año: en la época de lluvias, a finales de esa estación, y a mitad de la época de secas. Mensualmente, el valor más alto se registró en julio

Cuadro 1. Número de géneros, especies e individuos de las subfamilias de Staphylinidae, registradas en la Sierra de Huautla, Morelos, durante este estudio

\begin{tabular}{lccc}
\hline & Géneros & Especies & Individuos \\
\hline Aleocharinae & 15 & 25 & 1376 \\
Osoriinae & 1 & 1 & 2 \\
Oxytelinae & 7 & 14 & 11681 \\
Paederinae & 17 & 36 & 1440 \\
Piestinae & 1 & 1 & 5 \\
Scaphidiinae & 1 & 1 & 1 \\
Staphylininae & 4 & 7 & 112 \\
Steninae & 1 & 1 & 1 \\
Tachyporinae & 2 & 6 & 268 \\
\multicolumn{1}{c}{ Total } & 49 & 91 & 14886 \\
\hline
\end{tabular}

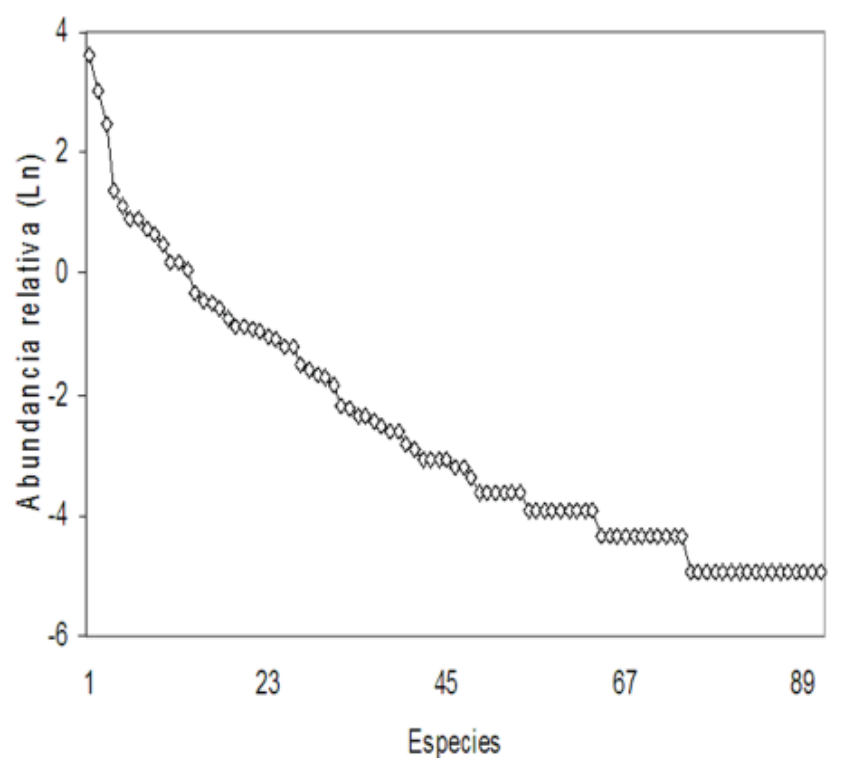

Figura 1. Patrón de abundancia relativa de las especies de Staphylinidae registradas en la Sierra de Huautla, Morelos durante este estudio.

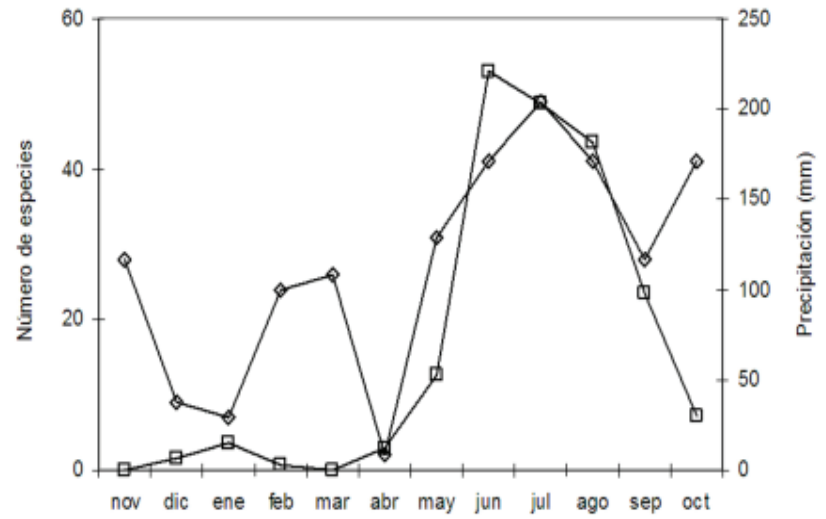

Figura 2. Patrón de riqueza (rombos) y precipitación (cuadros) mensual registrada en la Sierra de Huautla, Morelos durante este estudio.

y el valor más bajo en abril, justo al final de la época de sequía (Fig. 2).

En el caso de la abundancia, el patrón sí fue estacional; sus valores más altos se registraron en la época de lluvias y el más bajo en la época de secas (Fig. 3). Mensualmente, el valor más alto se registró en agosto y el más bajo en abril, justo al final de la época de sequía.

El mismo patrón anual de riqueza y abundancia también se observó al analizar cada una de las trampas de manera individual (Fig. 4 y 5), aunque con ligeras variaciones respecto al patrón general (Fig. 2 y 3). En las trampas 1 


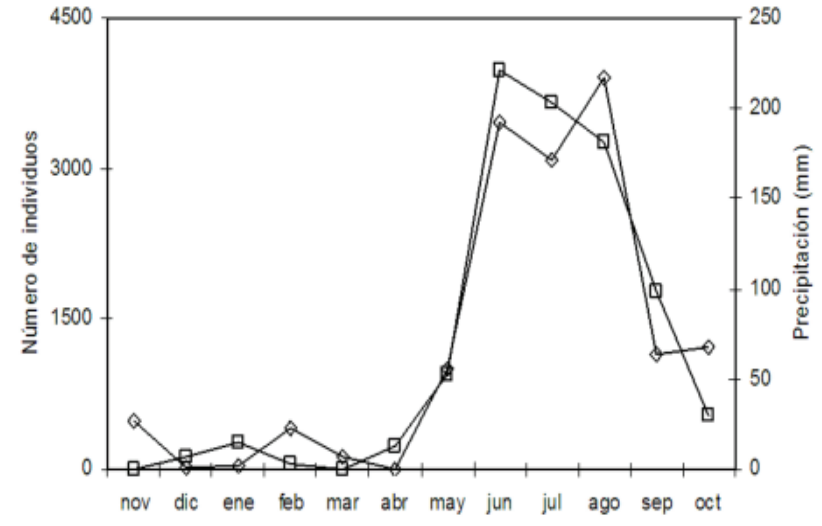

Figura 3. Patrón de abundancia (rombos) y precipitación (cuadros) mensual registrada en la Sierra de Huautla, Morelos durante este estudio.

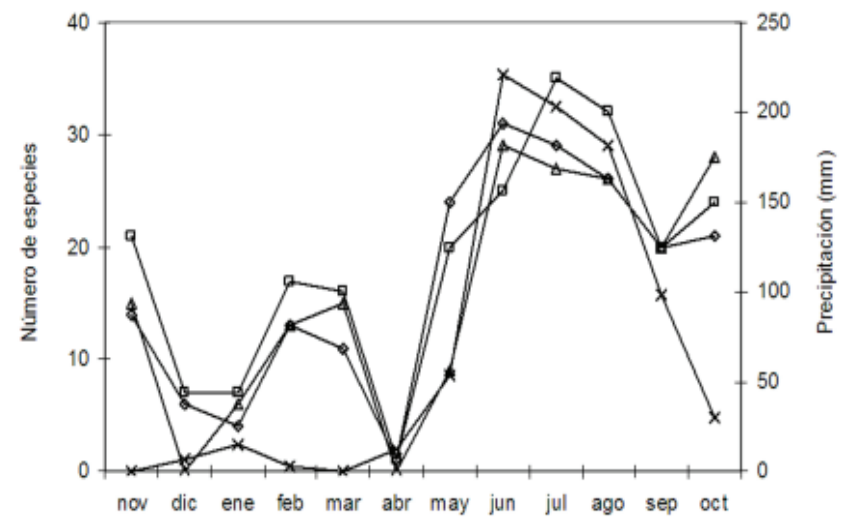

Figura 4. Patrón de riqueza y precipitación mensual registrada en la Sierra de Huautla, Morelos durante este estudio. Trampa $1=$ rombos, trampa $2=$ cuadros, trampa $3=$ triángulos, precipitación $=$ asteriscos.

y 3 la mayor riqueza se registró en junio (coincidiendo con el valor más alto de precipitación) y en la trampa 2 en julio; el incremento en la época de sequía se registró en febrero para las trampas 1 y 2 y en marzo para la 3 (Fig. 4). Respecto a la abundancia, ésta también fue estacional, registrándose el valor más alto para la trampa 1 en julio, para la 2 en agosto y para la 3 en junio (Fig. 5).

El patrón de abundancia registrado para toda la familia se observó también en las principales subfamilias de este grupo, excepto en Aleocharinae, donde el patrón no fue estacional y fue similar al registrado para la riqueza (Fig. 6). En Oxytelinae y Aleocharinae la mayor abundancia se registró en agosto, en Paederinae en julio, en Staphylininae en junio y en Tachyporinae en septiembre. Por el contrario, para estas subfamilias los valores más bajos se registraron en diciembre o enero y sólo Aleocharinae presentó un

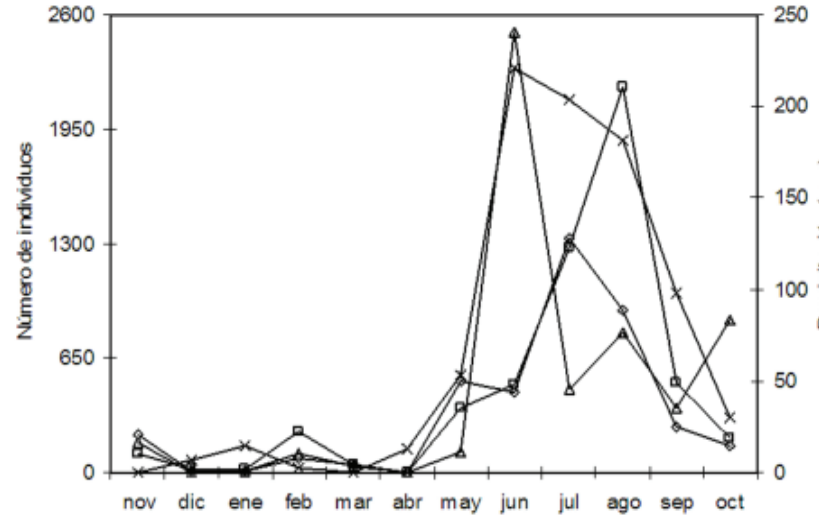

Figura 5. Patrón de abundancia y precipitación mensual registrada en la Sierra de Huautla, Morelos en cada una de las trampas utilizadas en el estudio. Trampa $1=$ rombos, trampa $2=$ cuadros, trampa $3=$ triángulos, precipitación $=$ asteriscos .

incremento importante en la época de secas (Fig. 6).

Estacionalmente, hubo diferencias en riqueza y abundancia entre la época de lluvias y de secas (MannWhitney U, p $=<0.0001$ para ambos casos) y en ambas variables los valores más altos se registraron en la época de lluvias. En este sentido y respecto a la abundancia, el $96 \%$ de los individuos se capturaron en la época de lluvias y sólo el $4 \%$ en la época de secas.

También hubo diferencias estacionales en riqueza y abundancia en las subfamilias más importantes (MannWhitney U, Aleocharinae $p=0.0007$, Oxytelinae $p=<0.001$ y Paederinae $p=<0.0001$ para riqueza y Aleocharinae $p=$ 0.0005 , Oxytelinae $p=<0.0001$ y Paederinae $p=<0.0001$ para abundancia) y en todos los casos, los valores más altos se registraron en la época de lluvias.

Respecto a la actividad por especie, 53 estuvieron activas exclusivamente en el periodo de lluvias, 5 sólo durante la época de secas y 33 en ambas estaciones. De estas últimas, la mayoría presentó su mayor abundancia en la época de lluvias, como Apocellus sp., Carpelimus sp. 1 y Platystethus sp. y sólo especies como Homalota sp. o Lathrobium sp. fueron también abundantes en la época de secas (Fig. 7).

Respecto a la actividad, con base en el número de meses en que fueron registradas, el $56 \%$ de las especies estuvieron activas un máximo de 2 meses, el $75 \%$ no más de 5 meses y sólo el $5 \%$ por más de 10 meses. Se encontró que más del $50 \%$ de las especies que tuvieron un periodo de actividad menor de 2 meses, están representadas por pocos individuos y conforme el número de individuos registrados es mayor, el tiempo de actividad registrado también se incrementa. En este sentido, hubo una relación significativa $\left(\mathrm{R}^{2}=0.2, p=<0.0001\right)$ entre la abundancia 

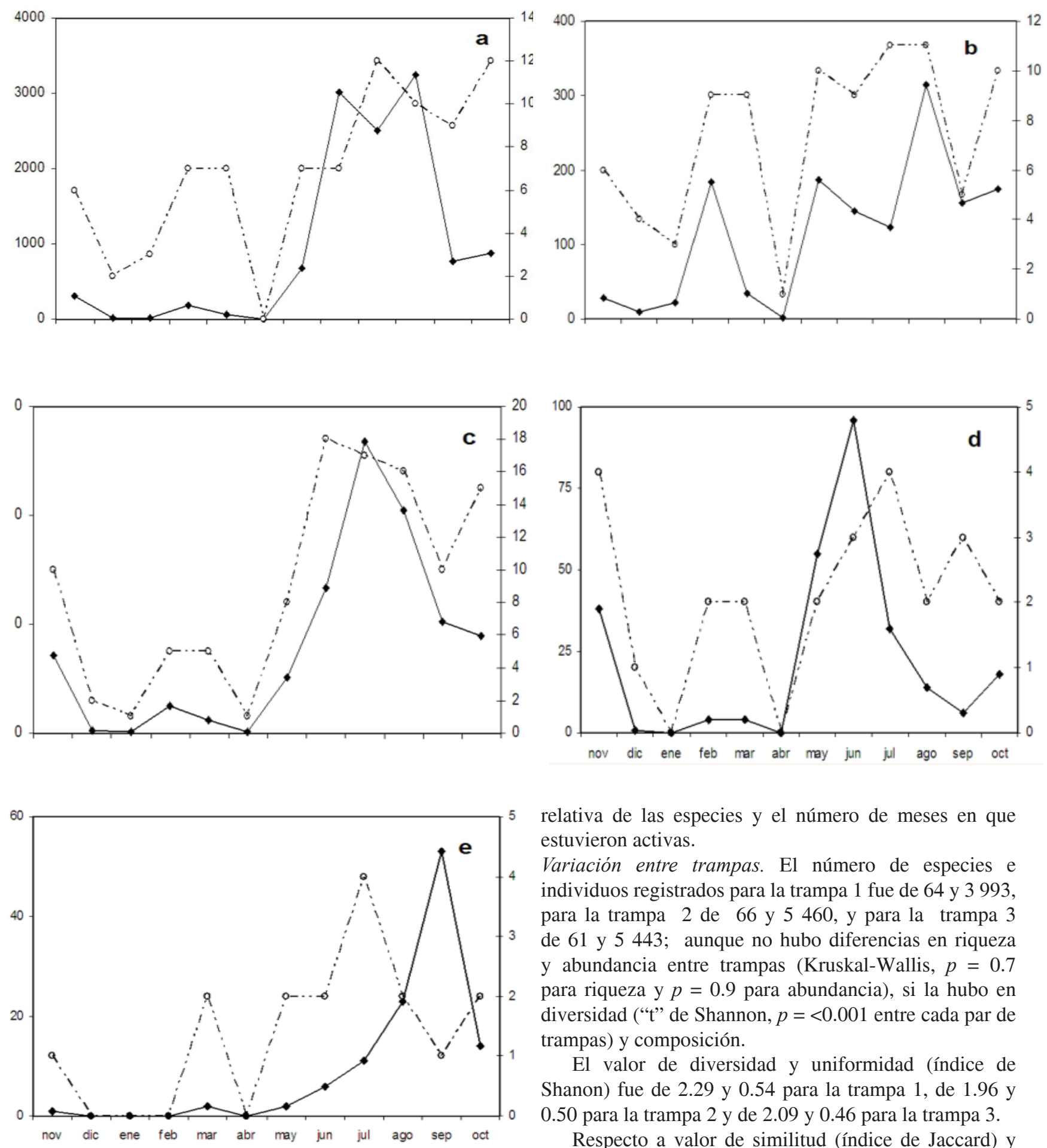

relativa de las especies y el número de meses en que estuvieron activas.

Variación entre trampas. El número de especies e individuos registrados para la trampa 1 fue de 64 y 3993 , para la trampa 2 de 66 y 5460 , y para la trampa 3 de 61 y 5 443; aunque no hubo diferencias en riqueza y abundancia entre trampas (Kruskal-Wallis, $p=0.7$ para riqueza y $p=0.9$ para abundancia), si la hubo en diversidad ("t" de Shannon, $p=<0.001$ entre cada par de trampas) y composición.

El valor de diversidad y uniformidad (índice de Shanon) fue de 2.29 y 0.54 para la trampa 1 , de 1.96 y 0.50 para la trampa 2 y de 2.09 y 0.46 para la trampa 3 .

Respecto a valor de similitud (índice de Jaccard) y número de especies compartidas entre trampas, fue del $62 \%$ y 49 especies entre las trampas 2 y 3 ; del $55 \%$ y 46 especies entre las trampas 1 y 2 , y del $51 \%$ y 42 especies entre las trampas 1 y 3 . En este mismo sentido, del total de especies, 13 se registraron sólo en la trampa 1; 8 en la trampa 2, y 7 en la trampa 3. Por el contrario, sólo

Figura 6. Patrón de riqueza (línea continua) y abundancia (línea discontinua) mensual registrada en la Sierra de Huautla, Morelos durante este estudio, para las subfamilias Oxytelinae (a), Aleocharinae (b), Paederinae (c), Staphylininae (d - rombos) y Tachyporinae (d - cuadros). Eje $\mathrm{Y}=$ Número de individuos, eje $\mathrm{YY}=$ número de especies. 

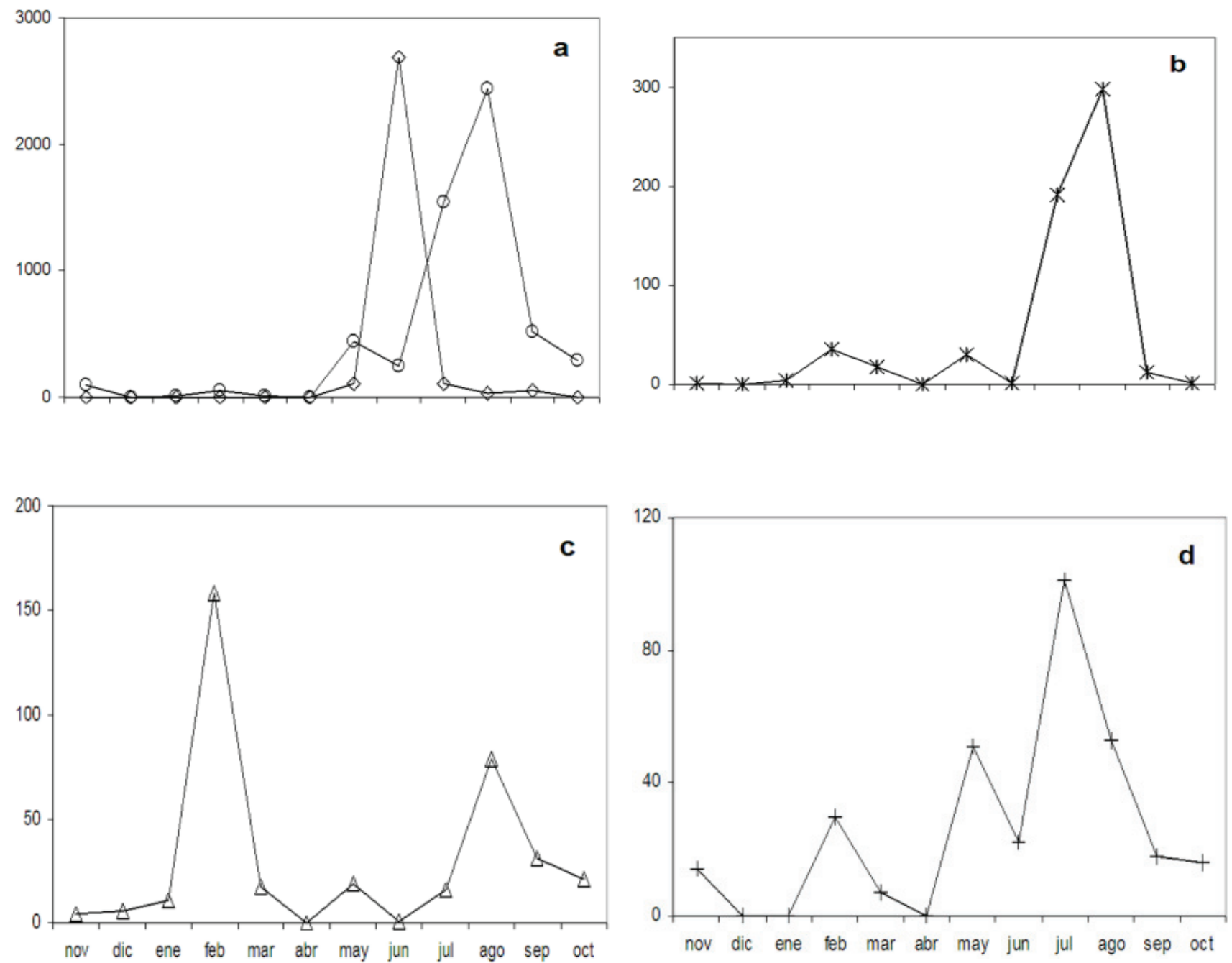

Figura 7. Patrón de abundancia mensual de las especies Apocellus sp. (a- rombos), Carpelimus sp. (a- círculos), Platystethus sp. (b), Homalota sp. (c) y Lathrobium sp. (d), registradas en la Sierra de Huautla, Morelos durante este estudio. Eje Y = Número de individuos.

se registraron 37 especies en las 3 trampas, 26 sólo en 2, y 28 sólo en 1 . Esto es, menos del $50 \%$ de las especies estuvieron representadas en las 3 trampas.

Al comparar la riqueza y abundancia entre trampas para las subfamilias más importantes (Aleocharinae, Oxytelinae y Paederinae), tampoco se encontraron diferencias en ambas variables, aunque hubo una mayor variación que al considerar la familia como un todo. En Oxytelinae, Aleocharinae y Paederinae hubo más especies en la trampa 2 , con 68,59 y 73 respectivamente, siguiéndole la 1 con 61,51 y 63 y la 3 con 56, 46 y 43 especies, habiendo una diferencia entre la primera y la última del 18, 22 y $41 \%$. En Tachyporinae hubo más especies en la trampa 3 con 16, siguiéndole la 2 con 15 y la 1 con 10 , lo que representa una diferencia del $37 \%$ entre la primera y la última. En
Staphylininae la mayor riqueza se registró en la trampa 3 con 25 especies, siguiéndole la trampa 1 con 11 y la 2 con 7 , lo que equivale a una diferencia del $72 \%$ entre las trampas 2 y 3 .

Respecto a la abundancia, las subfamilias Oxytelinae y Aleocharinae fueron más abundantes en la trampa 2 con 4300 y 539 individuos respectivamente, siguiéndole la 3 con 4427 y 463 y la 1 con 2954 y 374; con una diferencia del $31 \%$ entre la trampa 2 y la 1 en ambos casos. En Paederinae la mayor abundancia se registró en la trampa 2 con 525 individuos, le siguió la 1 con 518 y la 3 con 397 , lo que representa una diferencia del $24 \%$ entre la primera y la última. Tachyporinae tuvo una abundancia similar entre trampas $(92,74$ y 110 respectivamente), al igual que Staphylininae $(51,19$ y 42). 
Relación con variables climáticas. Tanto la abundancia como la riqueza estuvieron relacionadas con la precipitación $\left(\mathrm{R}^{2}=0.9, p=<0.0001 \mathrm{y} \mathrm{R}^{2}=0.7, p=0.0007\right.$ respectivamente), pero no con la temperatura.

\section{Discusión}

El patrón de actividad estacional registrado para la mayoría de las especies y su relación con la precipitación, es un patrón que ha sido documentado también para otros grupos de insectos en este tipo de vegetación (Noguera et al. 2002, 2007; Pescador et al., 2002; Zaragoza-Caballero et al., 2003). Este patrón parece estar relacionado a la época reproductiva y la disponibilidad de alimentos en el ecosistema, que en el bosque tropical caducifolio está regulado por la precipitación (Murphy y Lugo, 1986). Los valores más altos de productividad primaria neta se registran durante la época de lluvias (Martínez-Yrizar y Sarukhán, 1990) y por ende, la mayor cantidad de recursos alimenticios disponibles. Por lo tanto, la actividad reproductiva de las especies de estafilínidos y demás grupos de insectos activos durante este periodo, estaría sincronizada con el tiempo de mayor disponibilidad de recursos para el desarrollo de sus larvas.

No obstante, también hubo especies como Lathrobium sp. y Homalota sp., que estuvieron activas durante casi todo el año y que aparentemente no siguen el patrón estacional de la mayoría de las especies registradas durante el estudio. Esta falta de estacionalidad pudiera ser explicada por las preferencias de hábitat y los recursos alimenticios que estas especies explotan y verse afectada por las condiciones "artificiales" de humedad que el reservorio de agua provocan a escala local. En el caso de Lathrobium, los individuos viven en los detritos de hojarasca húmeda de hábitats riparios (Watrous, 1980) y en el caso de Homalota, debajo de la corteza de troncos caídos (Navarrete-Heredia et al., 2002). Aunque estos recursos están presentes todo el año en el BTC (Maass et al., 2002), el proceso de descomposición que permite que dicho recurso esté disponible para la comunidad heterotrófica está determinado por la precipitación, ya que durante la época de secas este proceso se detiene (Maass et al., 2002). En ese sentido, en los alrededores de la presa las condiciones de humedad permanecen más o menos estables a lo largo de todo el año, lo que podría estar determinando que dichos recursos continúen degradándose durante todo este tiempo y puedan ser utilizados por las especies de estafilínidos asociados con ellos. Esto permitiría que esas especies tuvieran más de una generación al año, como parecen mostrarlo los diferentes picos de abundancia registrados durante el periodo de estudio (Fig. 7c, d).
En contraste, durante la época de secas la humedad disminuye notablemente en el resto de la región y los diferentes cuerpos de agua ahí existentes se secan hasta el siguiente periodo de lluvias (Zaragoza-Caballero y Noguera, observación personal), probablemente deteniendo el proceso de degradación de la hojarasca y troncos muertos y provocando que este recurso no estuviera disponible en ese momento para los organismos heterótrofos. De ser así, fuera del área de influencia de la presa estas especies serían univoltinas y estarían presentes sólo en la época de lluvias.

Aunque lo anterior no puede probarse en este estudio, datos de otro grupo de insectos con requerimientos de hábitat similares parecen apoyar esta hipótesis. Algunas especies de Lampyridae fueron registradas durante $10 \mathrm{u} 11$ meses alrededor del mismo reservorio de agua; pero arroyo arriba, donde la humedad había desaparecido durante la época de secas, sólo se registraron en la época de lluvias (Zaragoza-Caballero et al., 2003).

Por otro lado, las diferencias en diversidad y composición registrada entre las trampas, a pesar de la distancia tan corta que hubo entre una y otra (menos de 300 $\mathrm{m}$ ), parece ser congruente con la gran heterogeneidad que caracteriza a este ecosistema (Murphy y Lugo 1995; Trejo y Dirzo, 2000; Durán et al., 2002). Se ha mostrado que a una escala local, variables ambientales como la altitud, pendiente, insolación y capacidad de retención de agua del suelo (todas asociadas a la disponibilidad de agua del suelo), explican cambios en la estructura y composición de la vegetación (Durán et al., 2002), lo que probablemente también afectaría la comunidad heterótrofa ahí existente. Para el caso de la región de estudio, la topografía es muy irregular y ello probablemente modifica las variables ambientales mencionadas arriba y da lugar a un mosaico de microhábitats con diferencias en estructura y composición de su flora y fauna. Al respecto, y corroborando la heterogeneidad registrada en este estudio, se ha encontrado que en escala local, la fauna de Staphylinidae se distribuye de forma muy heterogénea debido a variaciones de humedad, temperatura y perturbación del hábitat, no sólo en el BTC (Jiménez-Sánchez et al., 2001; Caballero, 2003) si no también en otro tipo de bosques (Navarrete-Heredia, 1996; Jiménez-Sánchez et al., 2001; Márquez, 2003). Además, esta heterogeneidad también se ha documentado a escala regional, dentro de un mismo tipo de hábitat (Anderson y Ashe, 2000).

\section{Agradecimientos}

Este estudio fue apoyado por el CONACYT a través de la beca otorgada a E. Jiménez-Sánchez y se vincula con el 
proyecto "Biodiversidad en Insecta [Odonata, Coleoptera (Cantharoidea, Cerambycidae), Diptera (Syrphidae), e Hymenoptera (Apoidea, Vespidae)] en 3 zonas del Pacífico Mexicano" auspiciado por el mismo CONACYT. Agradecemos su apoyo en el trabajo de campo a Enrique González Soriano, Enrique García Ramírez, Alicia Rodríguez Palafox ${ }^{\dagger}$, Beatriz Rodríguez Vélez, María Eugenia Guardado y Claudia Uribe Mu. También al Dr. J. L. Navarrete-Heredia de la Universidad de Guadalajara, Jalisco, México, por permitirnos consultar su colección y por su ayuda en la determinación de los especimenes, así como por sus valiosos comentarios, y a 2 revisores anónimos por sus sugerencias al manuscrito.

\section{Literatura citada}

Anderson, R. S. y J. S. Ashe. 2000. Leaf litter inhabiting beetles as surrogates for establishing priorities for conservation of selected tropical montane cloud forests in Honduras, Central America (Coleoptera: Staphylinidae, Curculionidae). Biodiversity and Conservation 9: 617-653.

Caballero, P. U. 2003. Staphylinidae (Insecta: Coleoptera) necrófilos de la Sierra de Huautla, Morelos, México. Tesis, Facultad de Estudios Superiores Iztacala, Universidad Nacional Autónoma de México, Tlalnepantla, Estado de México. 110 p.

Cervantes, S. L. 1988. Intercepción de lluvia por el dosel en una comunidad tropical. Ingeniería Hidráulica en México, II época 77: 5-17.

Chatzimanolis, S., J. S. Ashe y R. S. Hanley. 2004. Diurnal/ Nocturnalactivity of Rove Beetles(Coleoptera: Staphylinidae) on Barro Colorado Island Panama assayed by flight intercept trap. The Coleopterists Bulletin 58: 569-577.

CNA (Comisión Nacional del Agua). 2000. Datos climatológicos de la Estación Huautla. Comisión Nacional del Agua, Morelos, México.

Dorado, O. 1997. Sustainable development in the tropical deciduous forest of Mexico: Myths and realities. In Global genetic resources: access, ownership, and intellectual property rights, K. E. Hoagland y A. Y. Rossman (eds.). Association of Systematics Collections, USA. p. 263-278.

Dorado O., B. Maldonado, D. M. Arias, V. Sorani, R. Ramírez, E. Leyva y D. Valenzuela (elaboradores, redactores y compiladores). 2005. Programa de conservación y manejo Reserva de la Biosfera Sierra de Huautla. Comisión Nacional de Áreas Naturales Protegidas, SEMARNAT, México, D. F.

Durán, E., P. Balvanera, E. Lott, G. Segura, A. Pérez-Jiménez, Á. Islas y M. Franco. 2002. Estructura, composición y dinámica de la vegetación. In Historia Natural de Chamela, F. A. Noguera, J. H. Vega-Rivera, A. N. García-Aldrete y M. Quesada-Avendaño (eds.). Instituto de Biología, Universidad Nacional Autónoma de México, México, D .F. p. 443-472.

García, E. 1981. Modificaciones al sistema de clasificación climática de Köppen para adaptarlo a las condiciones de la
República Mexicana. Larios, México, D. F. 150 p.

Jiménez-Sánchez, E., J. L. Navarrete-Heredia y J. R. PadillaRamírez. 2001. Estafilínidos (Coleoptera: Staphylinidae) necrófilos de la Sierra de Nanchititla, Estado de México, México. Folia Entomológica Mexicana 108:53-78.

Jiménez-Sánchez, E., J. R. Padilla-Ramírez, S. StanfordCamargo y R. Quezada-García, 2001. Staphylindae (Insecta: Coleoptera) necrófilos de "El Salto de las Granadas", Guerrero, México. In Tópicos sobre Coleoptera de México, J. L. Navarrete-Heredia, H. E. Fierros-López y A. BurgosSolorio (eds.). Universidad de Guadalajara/Universidad Autónoma del Estado de Morelos, Jalisco/Cuernavaca. p. 55-68.

Kato, M., T. Inoue, A. A. Hamid, T. Nagamitsu, M. B. Merdek, A. R. Nona, T. Itino, S. Yamane y T. Yumoto. 1995. Seasonality and vertical structure of light-attracted insect communities in a dipterocarp forest in Sarawak. Researches on Population Ecology 37:59-79.

Klimaszewski, J. 2000. Diversity of rove beetles in Canada and Alaska (Coleoptera: Staphylinidae). Mémoires de la Société Royale Belge d'Entomologie, Bruxelles. 126 p.

Márquez, J. 2003. Ecological patterns in necrophilous Staphylinidae (Insecta: Coleoptera) from Tlayacapan Morelos, México. Acta Zoológica Mexicana (n.s.) 89:69-83.

Martínez-Yrizar, A. y J. Sarukhán. 1990. Litterfall patterns in a tropical deciduous forest in Mexico over a five-year period. Journal of Tropical Ecology 6:433-444.

Mass, J. M., V. Jaramillo, A. Martínez-Yrizar, F. García-Oliva, A. Pérez-Jiménez y J- Sarukhán. 2002. Aspectos funcionales del ecosistema de selva baja caducifolia en Chamela, Jalisco. In Historia Natural de Chamela, F. A. Noguera, J. H. VegaRivera, A. N. García-Aldrete y M. Quesada-Avendaño (eds.). Instituto de Biología, Universidad Nacional Autónoma de México, México, D. F. p. 525-542.

McAleece, N. 1997. BioDiversity, Professional. Version 2. http://www.sams.ac.uk/research/software. Consultado: 28 octubre 2000

Murphy, P. G. y A. E. Lugo, 1986. Ecology of tropical forest. Annual Review of Ecology and Systematic 17:67-88.

Murphy, P. G. y A. E. Lugo. 1995. Dry forests of Central America and the Caribbean. In Seasonally dry tropical forests, S. H. Bullock, H. A. Mooney y E. Medina (eds.). Cambridge University Press, Cambridge. p. 9-34.

Navarrete-Heredia, J. L. 1996. Coleópteros micetócolos de Basidiomycetes de San José de Los Laureles, Morelos, México. Tesis, Facultad de Ciencias, Universidad Nacional Autónoma de México. México, D. F. 179 p.

Navarrete-Heredia, J. L., A. F. Newton, M. K. Thayer, J. S. Ashe y D. S. Chandler. 2002. Guía ilustrada para los géneros de Staphylinidae (Coleoptera) de México. Ilustrated guide to the genera of Staphylinidae (Coleoptera) of México. Universidad de Guadalajara y CONABIO, México, D. F. 401 p.

Newton, A. F. y M. Thayer. 1992. Current classification and family-group names in Staphyliniformia (Coleoptera). Fieldiana Zoology (N.S.) 67:1-92.

Noguera, F. A., S. Zaragoza-Caballero, J. A. Chemsak, A. Rodríguez-Palafox, E. Ramírez, E. González-Soriano y R. Ayala. 2002. Diversity of the family Cerambycidae 
(Coleoptera) of the tropical dry forest of Mexico, I. Sierra de Huautla, Morelos. Annals of the Entomological Society of America 94:1-11.

Noguera, F. A., J. A. Chemsak, S. Zaragoza-Caballero, A. Rodríguez-Palafox, E. Ramírez-García, E. GonzálezSoriano y R. Ayala. 2007. A faunal study of Cerambycidae (Coleoptera) from one region with tropical dry forest in Mexico: San Buenaventura, Jalisco. The Pan-Pacific Entomologist 83:296-314.

Pérez-Jiménez, L. A., A. Flores-Castorena y G. Soria-Rocha. 1992. Clave para las familias de plantas con flores de la Sierra de Huautla, Morelos, México. Universidad, Ciencia y Tecnología 2:25-50.

Pescador-Rubio, A., A. Rodríguez-Palafox y F. A. Noguera. 2002. Diversidad y estacionalidad de Arthropoda. In Historia Natural de Chamela, F. A. Noguera, J. H. Vega-Rivera, A. N. García-Aldrete y M. Quesada-Avendaño (eds.) Instituto de Biología, Universidad Nacional Autónoma de México, México. p. 183-201.
Southwood, T. R. E. 1966. Ecological methods with particular reference to the study of insect population. Methuen, London.

Trejo, I. y R. Dirzo. 2000. Deforestation of seasonally dry tropical forest: a national and local analysis in Mexico. Biological Conservation 94:133-142.

Watrous, L. 1980. Lathrobium (Tetartopeus): natural history, phylogeny and revision of the neartic species (Coleoptera, Staphylinidae). Systematic Entomology 5:303-338.

Wolda, H. 1978. Fluctuations in abundance of tropical insects. The American Naturalist 112:1017-1045.

Zar, J. H. 1996. Biostatistical analysis, cuarta edición. Prentice Hall, Upper Saddle River, New Jersey. 663 p.

Zaragoza-Caballero, S., F. A. Noguera, J. A. Chemsak, E. González-Soriano, A. Rodríguez-Palafox, E. Ramírez-García y R. Ayala. 2003. Diversity of Lycidae, Phengodidae, Lampyridae, and Cantharidae (Coleoptera) in a tropical dry forest region in México: Sierra de Huautla, Morelos. The Pan-Pacific Entomologist 7:3-37.

Apéndice 1. Lista de especies de Staphylinidae registradas en la Sierra de Huautla, Morelos, durante este estudio. El listado incluye el número de individuos recolectados por especie. La clasificación taxonómica es de acuerdo a Newton y Thayer (1992).

\begin{tabular}{|c|c|c|c|c|c|c|c|c|c|c|c|c|c|}
\hline & Nov. & Dic. & Ene. & Feb. & Mar. & $A b r$ & May. & Jun. & Jul. & Ago. & Sep. & Oct. & Total \\
\hline \multicolumn{14}{|l|}{ Aleocharinae } \\
\hline Aleocharinae sp. & 0 & 0 & 0 & 0 & 0 & 0 & 0 & 0 & 1 & 0 & 0 & 0 & 1 \\
\hline \multicolumn{14}{|l|}{ Athetini } \\
\hline Atheta sp. 1 & 0 & 0 & 0 & 0 & 0 & 0 & 0 & 0 & 0 & 7 & 0 & 0 & 7 \\
\hline Atheta sp. 2 & 0 & 0 & 0 & 0 & 0 & 0 & 0 & 0 & 3 & 0 & 0 & 0 & 3 \\
\hline Atheta sp. 3 & 0 & 0 & 0 & 0 & 0 & 0 & 0 & 13 & 17 & 0 & 0 & 0 & 30 \\
\hline Atheta sp. 4 & 0 & 0 & 0 & 0 & 2 & 0 & 0 & 0 & 0 & 0 & 0 & 0 & 2 \\
\hline Atheta sp. 5 & 0 & 0 & 0 & 0 & 1 & 0 & 3 & 0 & 0 & 0 & 0 & 0 & 4 \\
\hline Atheta sp. 6 & 0 & 0 & 0 & 4 & 0 & 0 & 0 & 0 & 16 & 38 & 0 & 4 & 62 \\
\hline Atheta sp. 7 & 0 & 0 & 0 & 0 & 0 & 0 & 0 & 0 & 2 & 0 & 0 & 0 & 2 \\
\hline $\begin{array}{l}\text { Charoxus hermanii Kistner, } \\
1981\end{array}$ & 0 & 0 & 0 & 0 & 0 & 0 & 1 & 0 & 0 & 0 & 0 & 1 & 2 \\
\hline Charoxus sp. & 0 & 0 & 0 & 0 & 0 & 0 & 0 & 0 & 0 & 0 & 0 & 1 & 1 \\
\hline \multicolumn{14}{|l|}{ Deinopsini } \\
\hline Adinopsis sp. & 0 & 0 & 0 & 2 & 0 & 0 & 0 & 5 & 8 & 149 & 14 & 66 & 244 \\
\hline \multicolumn{14}{|l|}{ Homalotini } \\
\hline \multicolumn{14}{|l|}{ Gyrophaenina } \\
\hline Agaricomorpha sp. & 0 & 0 & 0 & 0 & 3 & 0 & 0 & 0 & 0 & 0 & 0 & 0 & 3 \\
\hline Probrachida sp. & 0 & 0 & 0 & 0 & 0 & 0 & 1 & 6 & 0 & 0 & 0 & 0 & 7 \\
\hline \multicolumn{14}{|l|}{ Homalotina } \\
\hline Homalota sp. & 4 & 6 & 11 & 158 & 17 & 0 & 19 & 1 & 16 & 79 & 31 & 21 & 363 \\
\hline \multicolumn{14}{|l|}{ Hoplandriini } \\
\hline Hoplandria sp. & 2 & 0 & 0 & 3 & 0 & 0 & 116 & 19 & 5 & 7 & 4 & 1 & 157 \\
\hline Tinotus sp. & 6 & 2 & 9 & 3 & 2 & 0 & 19 & 0 & 0 & 3 & 0 & 0 & 44 \\
\hline
\end{tabular}


Apéndice 1. Continúa

\begin{tabular}{|c|c|c|c|c|c|c|c|c|c|c|c|c|c|}
\hline & Nov. & Dic. & Ene. & Feb. & Mar. & $A b r$. & May. & Jun. & Jul. & Ago. & Sep. & Oct. & Total \\
\hline Tinotus sp. 2 & 0 & 0 & 0 & 4 & 0 & 0 & 0 & 0 & 0 & 0 & 0 & 0 & 4 \\
\hline \multicolumn{14}{|l|}{ Hypocyphtini } \\
\hline Oligota sp. & 0 & 0 & 0 & 3 & 1 & 0 & 0 & 0 & 0 & 3 & 0 & 0 & 7 \\
\hline \multicolumn{14}{|l|}{ Lomechusini } \\
\hline Tetradonia sp. & 0 & 0 & 0 & 0 & 0 & 0 & 0 & 0 & 0 & 0 & 0 & 1 & 1 \\
\hline Zyras sp. 1 & 0 & 1 & 0 & 1 & 6 & 0 & 13 & 2 & 0 & 6 & 4 & 0 & 33 \\
\hline Zyras sp. 2 & 2 & 0 & 0 & 0 & 1 & 0 & 0 & 0 & 0 & 0 & 0 & 0 & 3 \\
\hline Zyras sp. 3 & 0 & 0 & 0 & 0 & 0 & 0 & 0 & 0 & 1 & 2 & 0 & 0 & 3 \\
\hline \multicolumn{14}{|l|}{ Myllaenini } \\
\hline Myllaena sp. & 3 & 0 & 0 & 0 & 0 & 0 & 10 & 1 & 0 & 2 & 0 & 1 & 17 \\
\hline \multicolumn{14}{|l|}{ Oxypodini } \\
\hline \multicolumn{14}{|l|}{ Dinardina } \\
\hline Euthorax sp. & 11 & 1 & 2 & 5 & 0 & 0 & 2 & 94 & 52 & 18 & 102 & 78 & 365 \\
\hline \multicolumn{14}{|l|}{ Meoticina } \\
\hline Alisalia sp. & 0 & 0 & 0 & 0 & 2 & 1 & 2 & 3 & 2 & 0 & 0 & 1 & 11 \\
\hline \multicolumn{14}{|l|}{ OsORIINAE } \\
\hline Holotrochus sp. & 1 & 0 & 0 & 1 & 0 & 0 & 0 & 0 & 0 & 0 & 0 & 0 & 2 \\
\hline \multicolumn{14}{|l|}{ OXYTELINAE } \\
\hline \multicolumn{14}{|l|}{ Oxytelini } \\
\hline Anotylus sp. 1 & 0 & 0 & 0 & 7 & 2 & 0 & 12 & 0 & 1 & 0 & 0 & 5 & 27 \\
\hline Anotylus sp. 2 & 26 & 0 & 0 & 4 & 1 & 0 & 16 & 2 & 112 & 39 & 48 & 35 & 283 \\
\hline Apocellus sp. & 0 & 0 & 0 & 1 & 0 & 0 & 111 & 2684 & 113 & 32 & 59 & 3 & 3003 \\
\hline Platystethus sp & 1 & 0 & 4 & 35 & 18 & 0 & 30 & 1 & 191 & 298 & 12 & 2 & 592 \\
\hline \multicolumn{14}{|l|}{ Thinobiini } \\
\hline Bledius sp. 1 & 0 & 0 & 0 & 0 & 0 & 0 & 0 & 10 & 23 & 5 & 15 & 3 & 56 \\
\hline Bledius sp. 2 & 0 & 0 & 0 & 0 & 0 & 0 & 0 & 0 & 1 & 0 & 0 & 0 & 1 \\
\hline Carpelimus sp. 1 & 93 & 5 & 9 & 53 & 13 & 0 & 445 & 253 & 1543 & 2439 & 519 & 289 & 5661 \\
\hline Carpelimus sp. 2 & 189 & 6 & 4 & 84 & 16 & 0 & 48 & 41 & 501 & 422 & 111 & 383 & 1805 \\
\hline Carpelimus sp. 3 & 5 & 0 & 0 & 4 & 6 & 0 & 19 & 17 & 9 & 4 & 6 & 2 & 72 \\
\hline Thinobius sp. 1 & 0 & 0 & 0 & 0 & 1 & 0 & 0 & 0 & 0 & 0 & 0 & 2 & 3 \\
\hline Thinobius sp. 2 & 0 & 0 & 0 & 0 & 0 & 0 & 0 & 0 & 5 & 1 & 1 & 45 & 52 \\
\hline Thinobius sp. 3 & 1 & 0 & 0 & 0 & 0 & 0 & 0 & 0 & 7 & 4 & 1 & 97 & 110 \\
\hline Thinobius sp. 4 & 0 & 0 & 0 & 0 & 0 & 0 & 0 & 0 & 9 & 0 & 0 & 5 & 14 \\
\hline Thinodromus sp. & 0 & 0 & 0 & 0 & 0 & 0 & 0 & 0 & 0 & 2 & 0 & 0 & 2 \\
\hline \multicolumn{14}{|l|}{ Paederinae } \\
\hline \multicolumn{14}{|l|}{ Paederini } \\
\hline Acalophaena sp. 1 & 1 & 0 & 0 & 0 & 0 & 0 & 0 & 13 & 25 & 2 & 2 & 1 & 44 \\
\hline Achenomorphus sp. 1 & 0 & 0 & 0 & 0 & 0 & 0 & 0 & 1 & 6 & 0 & 2 & 5 & 14 \\
\hline Achenomorphus sp. 2 & 0 & 0 & 0 & 0 & 0 & 0 & 0 & 1 & 0 & 0 & 0 & 1 & 2 \\
\hline Astenus sp. 1 & 10 & 2 & 2 & 1 & 3 & 0 & 1 & 3 & 0 & 0 & 0 & 2 & 24 \\
\hline Astenus sp. 2 & 0 & 0 & 0 & 0 & 0 & 0 & 0 & 0 & 1 & 1 & 0 & 0 & 2 \\
\hline Biocrypta sp. 1 & 0 & 0 & 0 & 0 & 0 & 0 & 0 & 0 & 13 & 0 & 0 & 0 & 13 \\
\hline Biocrypta sp. 2 & 2 & 0 & 0 & 0 & 0 & 0 & 1 & 20 & 27 & 2 & 7 & 0 & 59 \\
\hline
\end{tabular}


Apéndice 1. Continúa

\begin{tabular}{|c|c|c|c|c|c|c|c|c|c|c|c|c|c|}
\hline & Nov. & Dic. & Ene. & Feb. & Mar. & $A b r$. & May. & Jun. & Jul. & Ago. & Sep. & Oct. & Total \\
\hline Complejo Medon sp. 1 & 0 & 0 & 0 & 0 & 1 & 0 & 0 & 0 & 0 & 0 & 0 & 0 & 1 \\
\hline Complejo Medon sp. 2 & 0 & 0 & 0 & 0 & 0 & 0 & 0 & 11 & 0 & 0 & 0 & 0 & 11 \\
\hline Echiaster sp. & 0 & 0 & 0 & 0 & 0 & 0 & 0 & 0 & 1 & 3 & 0 & 0 & 4 \\
\hline Eustilicus sp. & 0 & 0 & 0 & 0 & 0 & 0 & 0 & 0 & 0 & 0 & 0 & 1 & 1 \\
\hline Homaeotarsus sp. 1 & 0 & 0 & 0 & 0 & 0 & 0 & 0 & 0 & 1 & 4 & 1 & 0 & 6 \\
\hline Homaeotarsus sp. 2 & 0 & 0 & 0 & 0 & 0 & 0 & 0 & 47 & 3 & 1 & 0 & 0 & 51 \\
\hline Homaeotarsus sp. 3 & 0 & 0 & 0 & 0 & 0 & 0 & 0 & 3 & 0 & 0 & 0 & 1 & 4 \\
\hline Homaeotarsus sp. 4 & 0 & 0 & 0 & 0 & 0 & 1 & 0 & 3 & 72 & 4 & 1 & 4 & 85 \\
\hline Homaeotarsus sp. 5 & 0 & 0 & 0 & 0 & 0 & 0 & 0 & 1 & 0 & 0 & 0 & 0 & 1 \\
\hline Lathrobium sp. & 14 & 0 & 0 & 30 & 7 & 0 & 51 & 22 & 101 & 53 & 18 & 16 & 312 \\
\hline Lithocharis sp. 1 & 1 & 0 & 0 & 0 & 0 & 0 & 3 & 24 & 58 & 50 & 21 & 20 & 177 \\
\hline Lithocharis sp. 2 & 0 & 0 & 0 & 0 & 0 & 0 & 0 & 0 & 0 & 0 & 0 & 1 & 1 \\
\hline Lithocharis sp. 3 & 0 & 0 & 0 & 1 & 0 & 0 & 0 & 0 & 0 & 0 & 0 & 0 & 1 \\
\hline Lithocharis sp. 4 & 0 & 0 & 0 & 0 & 0 & 0 & 0 & 2 & 0 & 0 & 0 & 1 & 3 \\
\hline Lithocharis sp. 5 & 0 & 0 & 0 & 0 & 0 & 0 & 1 & 8 & 0 & 0 & 0 & 0 & 9 \\
\hline Lithocharis sp. 6 & 0 & 0 & 0 & 0 & 0 & 0 & 12 & 0 & 0 & 0 & 0 & 0 & 12 \\
\hline Monista sp. & 0 & 0 & 0 & 0 & 0 & 0 & 0 & 0 & 0 & 1 & 0 & 0 & 1 \\
\hline Ochthephilum sp. 1 & 0 & 0 & 0 & 0 & 0 & 0 & 0 & 2 & 0 & 0 & 0 & 0 & 2 \\
\hline Ochthephilum sp. 2 & 0 & 0 & 0 & 0 & 0 & 0 & 0 & 0 & 1 & 0 & 0 & 0 & 1 \\
\hline Orus sp. 1 & 0 & 0 & 0 & 1 & 0 & 0 & 0 & 0 & 0 & 6 & 0 & 0 & 7 \\
\hline Orus sp. 2 & 0 & 0 & 0 & 0 & 0 & 0 & 0 & 0 & 0 & 0 & 0 & 1 & 1 \\
\hline Paederus sp. 1 & 2 & 0 & 0 & 0 & 0 & 0 & 0 & 0 & 0 & 0 & 1 & 1 & 4 \\
\hline Paederus sp. 2 & 22 & 1 & 0 & 0 & 0 & 0 & 0 & 3 & 1 & 1 & 0 & 0 & 28 \\
\hline Scopaeus sp. 1 & 47 & 0 & 0 & 5 & 5 & 0 & 6 & 27 & 80 & 161 & 71 & 56 & 458 \\
\hline Scopaeus sp. 2 & 0 & 0 & 0 & 0 & 0 & 0 & 0 & 0 & 1 & 2 & 0 & 0 & 3 \\
\hline Scopaeus sp. 3 & 0 & 0 & 0 & 0 & 0 & 0 & 1 & 0 & 1 & 1 & 0 & 0 & 3 \\
\hline Thinocharis sp. 1 & 7 & 0 & 0 & 0 & 1 & 0 & 0 & 9 & 9 & 16 & 29 & 23 & 94 \\
\hline Thinocharis sp. 2 & 1 & 0 & 0 & 0 & 0 & 0 & 0 & 0 & 0 & 0 & 0 & 0 & 1 \\
\hline \multicolumn{14}{|l|}{ Piestinae } \\
\hline Piestus sp. & 0 & 0 & 0 & 0 & 2 & 0 & 1 & 1 & 1 & 0 & 0 & 0 & 5 \\
\hline \multicolumn{14}{|l|}{ Scaphidinae } \\
\hline Baeocera sp. & 0 & 0 & 0 & 0 & 0 & 0 & 0 & 1 & 0 & 0 & 0 & 0 & 1 \\
\hline
\end{tabular}

\section{Staphylininae}

\section{Staphylinini}

\section{Philonthina}

Neobisnius sp. 1

Neobisnius sp. 2

Philonthus sp. 1

Philonthus sp. 2

Philonthus sp. 3

\section{Quediina}

Heterothops sp

Xantholinini

Neohypnus sp. 1

$$
\begin{array}{ccccccccccccc}
0 & 0 & 0 & 0 & 0 & 0 & 0 & 5 & 3 & 19 & 53 & 12 & 92 \\
0 & 0 & 0 & 0 & 1 & 0 & 0 & 0 & 5 & 0 & 0 & 2 & 8 \\
0 & 0 & 0 & 0 & 0 & 0 & 0 & 0 & 2 & 4 & 0 & 0 & 6 \\
1 & 0 & 0 & 0 & 0 & 0 & 0 & 0 & 0 & 0 & 0 & 0 & 1 \\
0 & 0 & 0 & 0 & 0 & 0 & 0 & 1 & 0 & 0 & 0 & 0 & 1
\end{array}
$$

$\begin{array}{lllllllllllll}0 & 0 & 0 & 0 & 1 & 0 & 1 & 0 & 0 & 0 & 0 & 0 & 2\end{array}$

$\begin{array}{lllllllllllll}0 & 0 & 0 & 0 & 0 & 0 & 1 & 0 & 1 & 0 & 0 & 0 & 2\end{array}$


Apéndice 1. Continúa

\begin{tabular}{|c|c|c|c|c|c|c|c|c|c|c|c|c|c|}
\hline & Nov. & Dic. & Ene. & Feb. & Mar. & $A b r$. & May. & Jun. & Jul. & Ago. & Sep. & Oct. & Total \\
\hline \multicolumn{14}{|l|}{ Steninae } \\
\hline Stenus sp. & 0 & 0 & 0 & 0 & 0 & 0 & 1 & 0 & 0 & 0 & 0 & 0 & 1 \\
\hline \multicolumn{14}{|l|}{ TACHYPORINAE } \\
\hline \multicolumn{14}{|l|}{ Tachyporini } \\
\hline Coproporus hepaticus & 0 & 1 & 0 & 0 & 1 & 0 & 0 & 0 & 2 & 10 & 2 & 0 & 16 \\
\hline Coproporus sp. 1 & 1 & 0 & 0 & 1 & 0 & 0 & 0 & 0 & 0 & 0 & 0 & 0 & 2 \\
\hline Coproporus sp. 2 & 35 & 0 & 0 & 0 & 3 & 0 & 2 & 92 & 24 & 4 & 3 & 17 & 180 \\
\hline Coproporus sp. 3 & 1 & 0 & 0 & 3 & 0 & 0 & 53 & 1 & 4 & 0 & 1 & 0 & 63 \\
\hline Sepedophilus sp. 1 & 1 & 0 & 0 & 0 & 0 & 0 & 0 & 3 & 0 & 0 & 0 & 0 & 4 \\
\hline Sepedophilus sp. 2 & 0 & 0 & 0 & 0 & 0 & 0 & 0 & 0 & 2 & 0 & 0 & 1 & 3 \\
\hline
\end{tabular}

